Meta

Journal des traducteurs

Translators' Journal

\title{
La traduction technique
}

\section{Paul A. Horguelin}

Volume 11, numéro 1, mars 1966

URI : https://id.erudit.org/iderudit/003113ar

DOI : https://doi.org/10.7202/003113ar

Aller au sommaire du numéro

Éditeur(s)

Les Presses de l'Université de Montréal

ISSN

0026-0452 (imprimé)

1492-1421 (numérique)

Découvrir la revue

Citer cet article

Horguelin, P. A. (1966). La traduction technique. Meta, 11(1), 15-25.

https://doi.org/10.7202/003113ar

Ce document est protégé par la loi sur le droit d'auteur. L'utilisation des services d'Érudit (y compris la reproduction) est assujettie à sa politique d'utilisation que vous pouvez consulter en ligne.

https://apropos.erudit.org/fr/usagers/politique-dutilisation/ 


\section{la traduction technique}

C'est devenu une vérité de La Palice de dire que nous vivons au siècle de la technique et de la vitesse *. Il est peut-être plus intéressant de constater qu'il ne s'agit pas là d'un phénomène résultant d'une lente et longue évolution, mais la conséquence manifeste de ce qu'on a appelé le «processus d'accélération ». Il senable, en effet, qu'après avoir cheminé pendant des siècles et même des millénaires, le progrès scientifique, au début du $X X X^{e}$ siècle, a pris subitement le pas de course ou, pour user d'une comparaison empruntée au domaine technique, a changé d'engrenage, de palier. Je lisais dernièrement que la science a progressé davantage au cours des dix dernières années que depuis l'apparition de l'homo faber. Cette affirmation peut paraître exagérée, mais personne ne contestera, je crois, que depuis cinquante ans la science et la technique sont passées d'une progression arithmétique à une progression géométrique. Il suffit de penser, par exemple, au chemin qui sépare le biplan de 1914 de la fusée interplanétaire de 1964.

Parallèlement à ce progrès technique accéléré, on a assisté à une multiplication des échanges, rendus eux-mêmes possibles par l'application des découvertes scientifiques dans le domaine des moyens de communication et des techniques de diffusion. En supprimant la barrière des distances, l'avion, la radio, la télévision ont mis en présence des civilisations différentes, et donc des techniques différentes. En conséquence de l'accélération des progrès techniques, il est devenu nécessaire pour les entreprises privées comme pour les États de se tenir au courant des découvertes scientifiques et de leurs applications technologiques, s'ils ne veulent pas se laisser distancer; d'où la multiplication des organismes et congrès internationaux où les hommes de science échangent les résultats de leurs expériences, d'où aussi la prolifération des publications scientifiques et techniques grâce auxquelles chercheurs, ingénieurs et techniciens de tous les pays se tiennent informés des récents progrès et évitent de perdre un temps et des sommes considérables à refaire des travaux déjà faits.

* Exposé présenté devant les étudiants en traduction du Département de linguistique de l'Université de Montréal, le 11 décembre 1964. 
Depuis quelques années, donc, le volume des publications scientifiques et techniques a augmenté dans des proportions gigantesques en même temps que s'accroissaient les échanges entre pays. D'autre part, il est devenu indispensable pour les chercheurs et techniciens des secteurs privé et public de prendre rapidement connaissance du contenu de ces publications. Ces constatations sont confirmées par les statistiques: au cours des vingt dernières années, le nombre des revues scientifiques publiées dans le monde est passé de 24000 à plus de 60000 ; par ailleurs, le nombre des revues techniques russes traduites aux États-Unis a décuplé au lendemain du lancement du premier spoutnik. De la conjonction de ces faits - augmentation du volume des publications techniques et nécessité d'en connaître rapidement le contenu - sont nées ou se sont développées des professions et des spécialisations nouvelles, en particulier la profession de traducteur technique, de réviseur technique, d'ingénieur traducteur et celle, encore peu connue, de documentaliste linguiste.

Je me propose de vous parler d'abord de ces professions, de la formation qu'elles exigent, des débouchés qu'elles ouvrent, de l'avenir qui leur est réservé - en insistant, bien entendu, sur la profession de traducteur technique. Dans une seconde partie, je traiterai plus précisément du processus de la traduction technique pour tenter d'établir, à l'aide d'exemples pratiques, quelques règles sur la façon d'aborder et de résoudre les nombreux problèmes qui se posent au traducteur technique.

\section{Le traducteur technique}

Pendant longtemps, et surtout en Europe, qui disait traducteur pensait traducteur littéraire. Les choses ont bien changé, et la traduction technique dispute maintenant la première place aux «belles infidèles». Au Canada, la traduction littéraire a toujours occupé un rang relativement modeste, même si l'on constate depuis quelques années une nette augmentation des œuvres littéraires traduites par des traducteurs canadiens. La traduction technique n'a donc pour rivales que la traduction commerciale, rarement confiée à des traducteurs professionnels mais plus souvent à des secrétaires-traductrices, la traduction publicitaire et la traduction que $\mathrm{j}$ 'appellerais journalistique (agences de presse, journaux et magazines). Mais, en raison de l'omniprésence de la technique et de la science, le traducteur, quel que soit le domaine dans lequel il exerce son activité, est appelé à traduire des textes techniques au cours de sa carrière.

Qu'est-ce donc qu'un traducteur technique? Selon la définition donnée par le président de la Société française des traducteurs, le traducteur technique est un spécialiste de la profession dont la tâche est « d'assimiler un texte scientifique ou technique écrit dans une langue étrangère ... et de le réécrire de façon que le spécialiste auquel il est destiné ait l'impression qu'il a été écrit dans son propre pays ».

Pour accomplir cette tâche avec succès, le traducteur technique doit avoir reçu une double formation, l'une générale, l'autre spécialisée. Comme tout traducteur, le traducteur technique doit avoir une connaissance approfondie de la langue de départ et de la langue d'arrivée - connaissance des mots (lexique), 
de la structure de la phrase (agencement), du pays et des hommes (ethnographie et métalinguistique). Il est inutile d'insister sur cette première condition qui vous est bien connue. La seconde condition requise est la connaissance de la terminologie particulière des sciences et des techniques. Chaque science, chaque technique possède son vocabulaire ou son jargon qui permet aux spécialistes de mieux saisir la réalité de leur domaine propre. Au fur et à mesure que les sciences se spécialisent, les jargons deviennent plus nombreux et plus hermétiques. C'est ainsi que récemment il a fallu traduire en américain courant un traité scientifique rédigé en une langue incompréhensible pour les non initiés. D'autre part, la technique envahit peu à peu tous les domaines, et les hommes de science universels sont maintenant chose du passé. De ces deux constatations - existence d'une terminologie particulière à chaque technique et extension du domaine de la technique - découlent deux exigences propres à la traduction technique: la spécialisation et la documentation.

La spécialisation en traduction technique est une question délicate lorsqu'on l'envisage d'un point de vue pratique, car il faut tenir compte de plusieurs facteurs dont certains sont inconnus. Il y a d'abord la demande: il serait dangereux de se spécialiser dans une branche bien déterminée sans savoir à l'avance s'il y a des débouchés dans cette branche. Ensuite, la spécialisation ne doit pas être trop restreinte, sans quoi on risque de ne travailler qu'un mois par an ou d'attendre pendant dix ans le poste pour lequel on s'est préparé. Enfin, il y a le hasard qui, bien souvent, nous oriente dans une voie à laquelle on n'avait pas songé. Pour ces raisons, je crois qu'il est préférable de commencer par acquérir une culture technique et scientifique plus étendue que profonde, sur laquelle viendra se greffer par la suite la spécialisation. Et voici pourquoi: on ne peut demander à un. traducteur technique d'être une encyclopédie ambulante; dans la réalité, vous constaterez que c'est exactement ce qu'on attend de lui. Pour citer quelques exemples personnels, j'ai déjà eu à traduire des textes sur la technologie des plastiques, les machines électroniques, la pose de panneaux de revêtement, les allergies causées par les abeilles et, pour finir en beauté (si on peut dire), l'élevage des porcelets à l'engrais. Un traducteur technique doit être en mesure de faire face à la plupart de ces situations.

Les études du baccalauréat apportent déjà certaines bases, mais au niveau universitaire il est essentiel, à mon avis, d'y ajouter des connaissances plus spécialisées - notions de sciences appliquées, d'économie politique, de droit commercial et industriel, etc. - ne serait-ce que pour se familiariser avec chaque terminologie. On pourrait croire que le droit et la technique n'ont rien en commun; or, il arrive souvent qu'un texte technique se présente sous forme de soumission, contrat, marché, garantie ou brevet, et il faut évidemment traduire et les caractéristiques techniques et les clauses juridiques. Cette formation de base acquise, il est nécessaire de la compléter ensuite par des études plus spécialisées, des stages ou un perfectionnement autodidacte, et c'est à ce stade que devrait se situer la spécialisation.

Tout traducteur, quelle que soit sa. spécialité, doit se tenir documenté, ne serait-ce que pour être au courant de ce qui se passe dans le monde - et ainsi mieux comprendre et mieux rendre les textes qu'il a à traduire - et aussi 
pour enrichir et renouveler son vocabulaire. Mais pour le traducteur technique, la documentation est une nécessité absolue sans laquelle il ne peut espérer se tirer honorablement des difficultés les plus diverses et les plus inattendues qu'il a constamment à vaincre. Cette documentation se présente sous trois formes: la documentation écrite, les experts ou spécialistes, le fichier du traducteur.

Comme je l'ai dit, la traduction technique est un domaine illimité, et à quelques heures d'avis vous pouvez avoir à traduire un texte portant sur un sujet qui vous est totalement ou presque inconnu. Il est donc indispensable que vous puissiez rapidement (car en traduction, tout se fait rapidement) mobiliser toute l'aide possible pour mener à bien votre tâche. La première source à laquelle on pense est bien entendu la documentation écrite, c'est-à-dire dans l'ordre: les dictionnaires et glossaires techniques, les encyclopédies, les publications et les traités. Les dictionnaires techniques, encore plus que les dictionnaires de langue, sont souvent en retard de plusieurs années ou bien incomplets; or, fréquemment, les textes à traduire traitent de techniques ou d'applications récentes. Les glossaires sont généralement plus à jour et ils complètent avantageusement les dictionnaires, en particulier certains glossaires américains-français. Les encyclopédies sont utiles dans le cas de techniques qui ont peu évolué depuis quelques années (par exemple, la fabrication du caoutchouc synthétique ou le moteur à combustion interne); elles expliquent mais ne traduisent pas. Je rattache aux encyclopédies les livres de la collection «Que sais-je? », qui ont l'avantage de traiter de nombreux sujets, d'être relativement récents et d'un prix modique. Quant aux publications, il faut savoir que chaque technique, chaque profession a au moins un organe de diffusion; on ne peut évidemment les connaître ni les lire dans leur totalité, mais tout traducteur technique spécialisé devrait s'abonner aux revues qui touchent son domaine. Pour les traités enfin, leur consultation ou leur achat ne se justifie que si l'œuvre à traduire est assez importante. En résumé, le traducteur technique doit avoir sous la main un certain nombre d'outils, notamment plusieurs dictionnaires et glossaires techniques, et connaître en outre les principales sources de documentation — c'est le rôle du fichier auquel je ferai allusion tout à l'heure.

Lorsqu'un terme ou un passage n'est pas clair, ou lorsqu'on a épuisé sans succès toutes les sources de documentation, il faut avoir recours à l'expert ou au spécialiste. En traduction technique, la collaboration entre traducteur et expert est indispensable. Il y a deux genres d'experts, qui remplissent tous deux un rôle différent: tout d'abord, l'expert de la langue de départ, qui est le plus souvent l'auteur du texte. Il est le mieux qualifié pour expliciter sa pensée ou expliquer le sens d'un terme, et le traducteur ne doit pas hésiter à le consulter. Son rôle n'est pas de traduire, mais d'expliquer. En second licu, il y a l'expert de la langue d'arrivée qui, lui, peut fournir une traduction ou une explication, selon qu'il est bilingue ou non. Il faut toutefois prendre garde de ne pas accepter aveuglément les solutions proposées par l'expert, et ceci pour deux raisons: 1) l'expert n'est pas un linguiste, c'est un technicien, il ne faut donc pas lui demander plus qu'il ne peut donner; 2) il arrive souvent au Canada que l'expert soit de formation américaine ou qu'il travaille dans un milieu anglophone; le traducteur doit donc accepter avec circonspection les traductions proposées et les vérifier. 
Cette réserve faite, l'expérience m'a appris qu'il est très utile, sinon indispensable, d'avoir dans ses relations des spécialistes à qui l'on peut faire appel. Grâce à eux, et par un simple coup de téléphone, le traducteur s'épargne fréquemment des heures de recherches, parfois vaines.

Étant donné qu'en traduction le facteur «temps » est de première importance, le traducteur technique doit pouvoir rapidement localiser les sources de clocumentation; c'est ici que le fichier entre en jeu. Après la formation de base, la constitution d'un fichier devrait être le premier souci du traducteur technique. Ce fichier peut comprendre deux sections: l'une pour les références, l'autre pour le vocabulaire. Dans la première figure la liste des dictionnaires techniques et l'endroit où l'on peut les consulter, classés par ordre alphabétique selon les domaines particuliers; par exemple: Automobile, Électronique, Pétrole, etc. Comme point de départ, on peut se servir des fichiers déjà existants et des répertoires de dictionnaires (pour n'en citer que quelques-uns: le fichier des bibliothèques, la liste des dictionnaires techniques publiée dans le Journal des Traducteurs, le catalogue de l'A.T.S. ${ }^{1}$, la Bibliographie des dictionnaires scientifiques et techniques multilingues, publiée par l'UNESCO). Quant au fichier de vocabulaire, il ne se justifie, à mon avis, que si le traducteur technique se spécialise dans un ou quelques domaines, sinon il risque de mettre sur fiches une quantité de mots qu'il n'aura jamais plus l'occasion de rencontrer dans une traduction, ce qui est une perte de temps.

À ce fichier, j’ajouterais un classeur, également divisé en deux sections. Dans l'une, le traducteur garde dans des chemises classées une copie de toutes ses traductions, ce qui lui permet d'assurer une certaine uniformité de terminologie (la «consistency » chère à nos amis anglophones !) et, dans certains domaines (je pense en particulier à l'assurance), d'utiliser des passages de ses traductions précédentes. Dans l'autre, il accumule, également dans des chemises classées, la documentation recueillie sous forme d'articles, coupures de journaux, brochures ou prospectus, documentation qui s'enrichit au cours de ses lectures.

Avant de quitter le stade de la formation pour passer aux débouchés de la carrière, je désire ajouter que la formation du traducteur technique n'est jamais terminée: tous les jours il a de nouvelles difficultés à vaincre, et donc de nouvelles connaissances à acquérir. Il doit constamment se tenir au courant de l'actualité, pour accroître à la fois ses connaissances techniques et linguistiques. Comme son collègue le technicien, il ne peut se permettre de se laisser dépasser, il doit suivre la technique à la course.

La traduction technique n'est donc pas un métier de tout repos. Mais si on exige beaucoup du traducteur technique, il faut dire qu'en retour il n'est pas trop mal partagé. Après le rédacteur-traducteur publicitaire, le traducteur technique est probablement le mieux rémunéré et celui qui a le plus de facilité à se placer. Les débouchés dans ce domaine sont en effet très nombreux; en voici quelques exemples d'actualité: j'ai assisté récemment à deux conférences données chaque fois par le chef d'un service de traduction, et tous deux se sont plaints de la rareté des bons traducteurs techniques; la ville de Montréal a engagé

1. Associated Technical Service, Glen Ridge, New Jersey, U.S.A. 
dernièrement un traducteur pour la traduction de soumissions; la division de Montréal du Bureau fédéral de la traduction a embauché plusieurs traducteurs techniques et est à la recherche de réviseurs techniques; l'Expo 67 cherche de bons traducteurs techniques à la pige. Dans tous les cas on demande, en plus de la formation de traducteur, une formation technique ou juridique. Ces exemples se limitent au secteur public et à Montréal seulement. Il est évident que le secteur privé a également besoin de traducteurs techniques, et que ce besoin ira en grandissant pour les raisons que j'exposais tout à l'heure.

Voici donc ce qu'est le traducteur technique: un traducteur spécialisé à qui on demande une formation plus poussée que celle de son collègue non spécialisé, mais qui est aussi mieux rémunéré et voit s'ouvrir devant lui des débouchés toujours plus grands. Je voudrais maintenant dire quelques mots de trois professions connexes: celles de documentaliste linguiste, de réviseur technique et d'ingénieur-traducteur.

La profession de documentaliste linguiste est assez nouvelle et, à ma connaissance, elle a été définie pour la première fois en 1963 dans un bulletin de la Société française des traducteurs. En quelques mots ${ }^{2}$, le documentaliste linguiste est «chargé de la recherche et de l'exploitation de l'information». On le trouve dans le service de documentation des grandes entreprises, des ministères et des organismes internationaux; son rôle est de recueillir, classer, traduire ou faire traduire une masse d'informations pour ensuite choisir la documentation susceptible d'utilisation et la diriger vers les spécialistes qui en tireront le meilleur parti. Il semble que cette profession soit le résultat des deux phénomènes que je mentionnais au début, à savoir: l'accélération du progrès technique et l'augmentation du nombre des publications qu'il est nécessaire de déchiffrer rapidement. Toujours selon la Société française des traducteurs, « les offres d'emploi témoignent que l'on recherche plus de documentalistes linguistes que de simples traducteurs $\gg$.

La profession de réviseur est mieux connue; par contre, elle offre moins de débouchés que celle de traducteur technique, d'une part parce que le poste de réviseur est souvent offert à un traducteur chevronné en récompense de ses longs services; d'autre part, parce que seuls les bureaux de traduction assez importants ont des réviseurs attitrés. Le travail de réviseur est mieux rémunéré que celui de traducteur et, à mon point de vue, plus intéressant - sauf lorsqu'il est nécessaire de refaire la traduction, ce qui est une autre question.

Pour compléter ce bref tour d'horizon des professions se rattachant à la traduction technique, il faut mentionner un concurrent qui modifiera peut-être notre façon d'envisager la formation du traducteur technique; je veux parler de l'ingénieur-traducteur. À l'issue de la dernière guerre mondiale, les techniques et les machines américaines ont envahi l'Europe. Or, pour comprendre les techniques nouvelles et faire marcher les machines, il fallait traduire; comme il y avait une pénurie de traducteurs techniques, on a donné à des ingénieurs et à des techniciens une formation de traducteur. En France, les ingénieurs-traducteurs reçoivent un

2. Ceux que la question intéresse pourront se reporter à l'article de Paul Arnaud, "Le traducteur technique de l'avenir ... le documentaliste linguiste ", publié dans le Journal des Traducteurs, 9.3. 
traitement de $\$ 1,000$ par mois et ils sont très rares. Le technicien traducteur remplacera-t-il demain le traducteur technique ? c'est une question que l'on peut se poser ${ }^{3}$. Mais il est évident que la tentation est grande chez les employeurs de faire traduire des textes techniques par des techniciens plutôt que par des traducteurs. Les résultats ne sont pas toujours brillants; ils peuvent même être désastreux, comme j'ai déjà eu l'occasion de le constater. Une chose demeure évidente: que l'on soit traducteur technique ou ingénieur-traducteur, une double formation s'impose.

\section{Processus de la traduction technique}

Ce qui distingue la traduction technique des autres formes de traduction, et en constitue la plus grande difficulté, c'est le problème de compréhension. Il est évident que l'on ne peut traduire un mot ou même la description d'un procédé si on ne les comprend pas; or, en traduction technique, le cas se présente assez fréquemment, soit qu'il s'agisse d'un domaine ou d'une terminologie qui nous sont inconnus parce que trop spécialisés, soit que l'on ait affaire à une technique toute nouvelle dont le vocabulaire n'est pas encore fixé et encore moins enregistré dans les dictionnaires. Comme par ailleurs la traduction technique exige précision et exactitude, il ne peut être question d'éviter les difficultés ou de s'en tirer élégamment par un tour de passe-passe, comme cela est parfois possible dans d'autres domaines. Nous verrons tout à l'heure quelle est la marche à suivre pour résoudre les problèmes de compréhension. Auparavant, et pour procéder logiquement, commençons par la première phase du processus de traduction: l'analyse.

1. ANALYSE - L'analyse, qui est une opération importante en traduction littéraire, par exemple, se fait assez rapidement en traduction technique. Il est pourtant certains points qu'il ne faut pas négliger. Cette analyse peut se faire en quatre étapes, dont voici une brève description.

\section{1 - Lecture}

La lecture rend possible l'analyse; elle facilite la compréhension en donnant une vue d'ensemble sur le texte à traduire. Un terme qui semble équivoque au premier abord peut se trouver défini ou explicité dans un paragraphe suivant. Par exemple, le traducteur devra chercher dans le texte un indice qui lui indique s'il doit traduire repository for public funds par lieu de dépôt (emplacement) ou dépositaire (personne) des deniers publics. Le même cas se présente pour valve, comme on le verra plus loin.

3. Lors d'une récente séance d'étude du Cercle des traducteurs, la question a été effectivement posée par M. Robert Dubuc. Dans sa réponse, M. Santiago, chef du Service cle la traduction à l'Hydro-Québec, a fait ressortir qu'il serait difficile d'attirer des ingénieurs vers la traduction tant qu'on ne pourrait pas leur offrir un traitement au moins égal à celui d'ingénieur. Tout en regrettant cet état de choses, le conférencier a exprimé l'avis que l'ingénieur-traducteur était la solution d'avenir. 


\section{$1.2-$ Contexte}

Le contexte, pris au sens large du terme, permet de répondre à deux questions importantes: 1) Quelle est l'origine et la destination du texte à traduire ? s'agit-il d'un texte purement scientifique ( $p$. ex. communication à un congrès de physiciens) ou d'un ouvrage de vulgarisation destiné au grand public ? La réponse à cette question influera sur le niveau de langue. 2) Le texte est-il rédigé en anglais ou en américain? Cette question est déterminante sur le plan sémantique (surtout dans le domaine de la technique). Le traducteur des Mémoires de Churchill ne se l'est sans doute pas posée lorsqu'il traduit le mot truck, puisqu'il a vu un convoi de camions là où il y avait une rame de wagons ${ }^{4}$. De même, les corporate stocks sont des emprunts de municipalités en Grande-Bretagne et des titres de sociétés commerciales aux États-Unis.

\section{3 - Niveau et tonalité}

Selon la destination du texte, le niveau pourra être celui du jargon technique, de la langue écrite scientifique ou de la langue commune parsemée de termes techniques. La tonalité est en général neutre, impersonnelle.

\section{4 - Documentation}

Parvenu à ce stade de l'analyse, le traducteur est en mesure de déterminer le genre de documentation dont il aura besoin. Il peut déjà vérifier ses sources de documentation, faire l'inventaire des outils qu'il a sous la main et de ceux qu'il devra se procurer. Si l'importance de la traduction le justifie, il peut commencer à lire des traités pertinents, au double point de vue de la terminologie et de l'information technique.

2. COMPRÉHENSION - Le processus de traduction en général comporte une double opération: traduire pour comprendre et traduire pour faire comprendre. Dans le premier cas, le traducteur est seul en présence du texte; dans le second, le lecteur entre en jeu. En pratique, ces deux opérations sont simultanées, mais en traduction technique il est bon de les dissocier parce que, comme nous l'avons dit, la compréhension présente de plus grandes difficultés que dans les autres formes de traduction. On peut résoudre les problèmes de compréhension par étapes successives.

\section{1 - Degré de difficulté}

Le traducteur procède à un inventaire rapide des difficultés de compréhension pour déterminer $a$ ) s'il a la compétence voulue pour traduire: compréhension suffisante; documentation et experts à sa disposition; $b$ ) s'il peut traduire rapidement et de façon rentable, c'est-à-dire dans le délai imparti et dans un temps correspondant à la rémunération.

4. Je dois cet exemple à M. J.-P. Vinay. 


\section{2-- Genre de difficulté}

Au cours de la lecture, le traducteur a relevé les termes et les passages qu'il ne comprend pas. Des recherches préliminaires à l'aide des outils et de la documentation dont il dispose lui permettent d'éliminer les difficultés qu'il peut résoudre seul.

\section{3 - Appel à l'expert}

S'il reste des termes ou des passages obscurs ou incompréhensibles, le traducteur doit faire appel à l'expert. Le temps de celui-ci étant précieux, il est important de lui poser des questions précises et intelligentes, de préférence avec renvoi au texte.

\section{4 - Traitement des réponses}

Lorsqu'on reçoit les réponses, deux cas peuvent se présenter: ou bien les explications permettent de traduire ou bien, tout en comprenant le sens, on ne connaitt pas l'équivalent dans la langue d'arrivée. En fait, il ne s'agit déjà plus d'un problème de compréhension mais de traduction, et nous retrouverons les réponses, sous forme d'exemples, à la $3^{\mathrm{e}}$ phase du processus qui est la traduction proprement dite.

3. TRADUCTION - Il s'agit maintenant de traduire pour faire comprendre. À ce stade, le traducteur comprend dans sa totalité le texte dans la langue de départ, il a sous la main les outils nécessaires pour travailler et les explications que lui a fournies l'expert sur les points obscurs. Il ne reste donc plus qu'à passer à la traduction, c'est-à-dire à la recherche d'équivalences. Voici quelques exemples pratiques dont l'intérêt réside davantage dans la manière d'arriver à la solution que dans la solution elle-même.

$1^{\text {er }}$ cas: Le terme est inconnu ou il est employé dans un sens inconnu. L'explication de l'expert de la LD permet de comprendre et de traduire:

CASTER

IRON PIPE NPT

$10^{\prime \prime}$ O.D.

PERFORATED TYPE BREAKWATER
Contexte: Liste de produits fabriqués par une société. Dictionnaire (Harrap): 1) mouleur, fondeur; 2) déschisteur.

Explication: Petite roue placée sous une plate-forme ou un chariot.

Traduction: roulette (pivot et roulement à billes)

Simple problème de compréhension.

Les abréviations, fréquentes dans les tableaux, les plans, les croquis cotés, etc., sont souvent des énigmes que l'expert de la LD permet de résoudre sans difficulté. Ici: National Pipe Thread - Outside Diameter.

Cet exemple (cité par M. P. Griffon, réviseur au Bureau fédéral de la traduction à Montréal) illustre les dangers de la traduction littérale. Il ne s'agissait pas d'un brise-lames perforé, comme devait l'expliquer l'expert, mais d'un briselames alvéolé. 
$2^{e}$ cas: Termes imprécis ou équivoques. Beaucoup de mots n'ont pas la même extension dans les deux langues; d'autres ont plusieurs équivalents se situant à des niveaux de langue différents. Dans chaque cas, le traducteur doit rechercher le terme précis, technique, et demander des précisions à l'expert si le contexte n'est pas assez clair. Voici deux exemples:

VALVE

Équivalents possibles: robinet, soupape, vanne, valve. Mais ces mots ne sont pas synonymes, et le traducteur devra se reporter au contexte ou faire appel à l'expert pour s'assurer qu'il emploie le terme exact. La pose d'une vanne à la place d'une soupape peut avoir des conséquences fâcheuses !

SCREEN

Ce mot peut désigner, entre autres choses, un grillage, mais dans un texte technique la précision exige que l'on traduise, selon le cas, par tamis, claie, crible, sas, etc. De même flyscreen ne devra pas se rendre par moustiquaire, mais par toile métallique (tout comme cheese-cloth n'est pas du « coton à fromage $\gg$ mais de la gaze !)

$3^{e}$ cas: Dans les cas qui précèdent, grâce au contexte ou aux explications de l'expert de la LD, il a été possible de trouver des équivalences satisfaisantes. Mais il n'en est pas toujours ainsi, et parfois il faut avoir recours à l'expert de la LA. Voici un exemple qui permet de passer en revue chacune des étapes de la démarche:

ROUTINE \& SUB-ROUTINE
Contexte: Il s'agit de machines électroniques, mais les deux termes figurent dans une liste, sans autres explications.

Dictionnaires: Les dictionnaires unilingues ou bilingues ne donnent pas le sens technique des deux termes.

Expert de la $L D$ : en l'occurrence, c'est le client qui a fourni le texte à traduire. Son explication est la suivante: Série d'instructions données à une machine électronique pour remplir une fonction déterminée 1) qui s'applique à presque tous les cas (routine) ou 2) seulement à quelques cas (subroutine). Ex.: 10000 factures à imprimer, 50 avec taxe municipale. Routine: calcule les prix et imprime. $S u b$ routine: calcule et ajoute la taxe.

Le problème de compréhension est résolu, mais non celui de la traduction. La recherche d'équivalences dans les ouvrages de documentation n'apporte aucune solution satisfaisante. Il faut donc passer à la dernière étape.

Expert de la $L A$ : Dans le cas présent, je me suis adressé au Centre de calcul de l'Université de Montréal. On m'a d'abord proposé «routine» et «sous-routine», solution de facilité que j'accueille avec une grimace! Après discussion et consultation de glossaires, nous arrivons à la solution: programme et sous-programme. La traduction me satisfait et je l'adopte ... tout en espérant n'avoir jamais à traduire " program and routine ». À chaque jour suffit sa peine! 
4. RÉVISION - En règle générale, il est toujours avantageux de faire réviser sa traduction. La réaction du lecteur qui ne connaît pas l'original en LD est un excellent critère de «transparence ». En traduction technique, toutefois, la révision doit porter davantage sur le fond que sur la forme, la précision et l'exactitude passant avant l'élégance. C'est pourquoi la révision devrait être confiée à un technicien, qui sera mieux en mesure de déceler rapidement les termes inexacts et de signaler au traducteur les passages qui manquent de clarté. Le but primordial de la traduction technique étant l'information, il est évidemment essentiel que le message soit clair.

De ce qui précède on peut conclure que la traduction technique est un travail sinon d'équipe, du moins de collaboration étroite entre le traducteur et le technicien. Sans cette collaboration, il est difficile - et parfois impossible - de faire ce travail de façon satisfaisante. C'est une profession qui exige à la fois un esprit curieux, car le traducteur doit se tenir au courant des progrès de la technique et accroître sans cesse ses connaissances dans un grand nombre de domaines; un esprit méthodique, car il doit classer et répertorier toute la documentation qu'il accumule pour pouvoir l'utiliser au moment voulu sans perte de temps; enfin, un souci de l'exactitude et de la précision, car la science et la technique ne se contentent pas d'à-peu-près. Sur le plan linguistique, le traducteur technique est souvent appelé en outre à faire œuvre de pionnier. Dans sa chronique «Défense de la langue française », Robert Le Bidois écrivait récemment: "C'est surtout dans le domaine de la technique et de la science qu'apparaissent les insuffisances du vocabulaire. Tous les jours, de nouveaux concepts, dispositifs ou objets nous obligent à créer des mots qui ne figurent pas dans les dictionnaires. $\gg$ Or, c'est au traducteur technique qu'il incombe souvent de combler ces lacunes lexicales. La traduction technique offre donc à ceux qui ont la compétence et les dispositions voulues une carrière à la fois enrichissante pour l'esprit, rémunératrice et pleine d'avenir.

Paul A. Horguelin

Montréal 